\title{
Leucostase secundária a leucemia mieloide aguda, com manifestação de síndrome respiratória aguda: relato de caso
}

\author{
Leukostasis secondary to acute myeloid leukemia, with manifestation of acute respiratory \\ syndrome: case report
}

\section{Leucostasis secundaria a leucemia mieloide aguda, con manifestación de síndrome respiratorio agudo: reporte de caso}

Soraya Souto da Silva ${ }^{1 *}$, Wilson de Oliveira Filho ${ }^{1}$, Ketllyn de Oliveira Cruz ${ }^{1}$, Bruna Lyandra Portela Sena de Souza1', Gabriela Lopes Araújo'.

\section{RESUMO}

Objetivo: Relatar um caso de uma paciente diagnosticada em julho de 2019 com leucostase secundária a leucemia mieloide aguda, com manifestação de síndrome respiratória aguda. Detalhamento de caso: Paciente procedente de Manaus-AM, com 70 anos, apresentando um quadro de dor abdominal e desconforto torácico importante de início há três meses, procurando serviço de urgência por três vezes e sendo diagnosticada com fibrilação atrial. Constatada leucocitose importante (> 200.000) em exames em departamento de urgência. Internada para investigação diagnóstica. A paciente só obteve melhora do quadro clínico após a realização da citorredução com hidroxiureia, com diminuição da contagem de leucócitos para 50.000. Com isso, chegou-se ao diagnóstico clínico de leucostase, secundária a leucemia mieloide aguda, com comprometimento pulmonar importante e neurológico leve. Considerações finais: $O$ acompanhamento desse caso permitiu observar a complexidade do quadro clínico desenvolvido pela paciente e a importância do diagnóstico precoce e instauração do tratamento adequado, evitando que os sintomas se agravassem ainda mais, colocando em risco a vida da paciente.

Palavras-chave: Leucemia mieloide aguda, Leucostase, Síndrome respiratória aguda.

\section{ABSTRACT}

Objective: To report a case of a patient diagnosed in July 2019 with leukostasis secondary to acute myeloid leukemia, with manifestation of acute respiratory syndrome. Case details: Patient from Manaus-AM, 70 years old, presenting with abdominal pain and significant chest discomfort beginning three months ago, seeking emergency service three times, and being diagnosed with atrial fibrillation. Important leukocytosis (> 200,000 ) was found in exams in the emergency department. Interned for diagnostic investigation. The patient only improved her clinical condition after performing cytoreduction with hydroxyurea, with a decrease in the leukocyte count to 50,000 . With that, the clinical diagnosis of leukostasis, secondary to acute myeloid leukemia, was reached, with significant pulmonary and mild neurological impairment. Final considerations: The follow-up of this case allowed us to observe the complexity of the clinical picture developed by the patient and the importance of early diagnosis and the establishment of adequate treatment, preventing the symptoms from getting worse, putting the patient's life at risk.

Keywords: Acute myeloid leukemia, Leukostasis, Acute respiratory syndrome.

\section{RESUMEN}

Objetivo: Reportar el caso de una paciente diagnosticada en julio de 2019 con leucoestasis secundaria a leucemia mieloide aguda, con manifestación de síndrome respiratorio agudo. Detalle del caso: Paciente de Manaus-AM, 70 años, que consulta con dolor abdominal y malestar torácico significativo desde hace tres meses, que acude a urgencias en tres ocasiones y se le diagnostica fibrilación auricular. Se encontró leucocitosis importante (> 200.000) en los exámenes en el servicio de urgencias. Internado para investigación diagnóstica. La paciente solo mejoró su estado clínico tras realizar citorreducción con hidroxiurea, con descenso del recuento leucocitario a 50.000. Con ello se llegó al diagnóstico clínico de

\footnotetext{
${ }^{1}$ Hospital Universitário Getúlio Vargas, Manaus - AM. *E-mail: sorayasouto@gmail.com
} 
leucostasis, secundaria a leucemia mieloide aguda, con importante afectación pulmonar y neurológica leve. Consideraciones finales: El seguimiento de este caso permitió observar la complejidad del cuadro clínico desarrollado por el paciente y la importancia del diagnóstico precoz y la implementación de un tratamiento adecuado, evitando que los síntomas empeoren, poniendo en riesgo la vida del paciente.

Palabras clave: Leucemia mieloide aguda. Leucoestasis, Síndrome respiratorio agudo.

\section{INTRODUÇÃO}

Casos de leucemia mieloide aguda (LMA) podem se manifestar com hiperleucocitose (HL), que é definida como uma contagem de leucócitos (> $100 \times 10^{9} / \mathrm{L}$.), levando a leucostase, síndrome de lise tumoral (SLT) e/ou coagulação intravascular disseminada (CID). Leucostase refere-se a complicações do órgão-alvo devido a leucoagregados microvasculares, hiperviscosidade, isquemia tecidual, infarto e hemorragia que não são atribuíveis a etiologias infecciosas, tromboembólicas ou outras subjacentes. O desenvolvimento da leucostase depende do número de leucócitos e de fatores como diferentes características morfológicas, moleculares e de plasticidade das células blásticas, bem como da capacidade das células endoteliais de liberar as citocinas (TENDULKAR AA, et al., 2017).

A HL está presente em $10 \%$ a $20 \%$ dos pacientes com LMA recentemente diagnosticada, contudo, os sintomas de leucostase ocorrem com menos frequência e geralmente afetam pacientes com contagem de leucócitos> $100 \times 10 \%$ L. Além disso, os pacientes com LMA e HL apresentam alto risco de mortalidade precoce como resultado de complicações pulmonares, renais e do sistema nervoso central (PIRO E, et al., 2011).

A HL apresenta alta morbidade e mortalidade precoce por complicações relacionadas à leucostase, principalmente hemorragia intracraniana e desconforto pulmonar (THAPA N, et al., 2018). A leucostase pode causar várias complicações, incluindo síndrome de hiperviscosidade, oclusão vascular resultando em hemorragias intracranianas e insuficiência respiratória e infiltrados leucêmicos perivasculares (AQUI N, O'DOHERTY U, 2014).

Pacientes com LMA tambémpodem apresentar várias complicações pulmonares durante o curso de sua doença. Essas complicações podem ser causadas não apenas por infecções usuais ou oportunistas, mas também por lesões pulmonares não infecciosas relacionadas diretamente ao processo leucêmico ou ao seu tratamento (MOREAU AS, et al., 2014).

Em um estudo prospectivo, realizado por Chaoui D, et al. (2004), com 65 adultos, a maioria das complicações pulmonares precoces em pacientes com LMA foram devido a causas não infecciosas. Segundo Moreau AS, et al. (2014), antes da quimioterapia de indução, a leucostase e a infiltração leucêmica pulmonar são os dois mecanismos específicos da leucemia que podem causar eventos respiratórios. Ainda de acordo com estes autores, a leucostase pode ocorrer quando a contagem de células blásticas periféricas é muito alta ou aumenta rapidamente, levando a lesão endotelial e ativação por invasão microvascular, hiperviscosidade, microtrombos leucocíticos e sequestro de oxigênio.

O manejo eficaz das manifestações respiratórias na fase inicial das hemopatias mieloides agudas, especialmente a LMA, frequentemente requer uma estreita colaboração entre hematologistas, pneumologistas e intensivistas.Dominadas por etiologias infecciosas, existem, no entanto, entidades patológicas específicas que não devem ser negligenciadas na abordagem diagnóstica e terapêutica.Estes incluem leucostase pulmonar, infiltração pulmonar leucêmica, pneumopatia por lise celular e proteinose alveolar secundária (VINCENT F, et al., 2012).

Conforme explicam Bewersdorf JP e Zeidan AM (2020), as interações entre blastos leucêmicos e células endoteliais que levam à leucostase estão se tornando cada vez mais compreendidos. Os blastos leucêmicos interagem com as células endoteliais por meio de moléculas de adesão celular. E na ausência de terapias direcionadas aprovadas, a hidroxiureia permanecem a base da terapia para pacientes com LMA e hiperleucocitose. 
Esse estudo teve o objetivo de apresentar um relato de caso de paciente com leucostase secundária a leucemia mieloide aguda, que apresentou manifestação de síndrome respiratória aguda, nointuito de acrescentar dados sobre o diagnóstico e o tratamento desse caso.

\section{DETALHAMENTO DO CASO}

Em Manaus, no Amazonas (AM), em 26 de julho de 2019, M.G.S.A, paciente do sexo feminino, com 70 anos, foi admitida na enfermaria de hospital local, apresentando "dor no peito" como queixa principal. Em anamnese constatou-se um quadro de dor abdominal e desconforto torácico importante de início há três meses, procurando serviço de urgência por três vezes e sendo realizado holter em 24 de junho de 2019, cujo laudo pode ser visto no Quadro 1, diagnosticada com alterações cardíacas em 26 de junho do mesmo ano (Tabela 1) e fibrilação atrial em 10 de julho do mesmo ano (Figura 1) iniciando tratamento com Xarelto ${ }^{\circledR}$ (Rivaroxabana, fármaco anticoagulante oral que age como inibidor direto do fator $\mathrm{Xa}$ ), $20 \mathrm{mg} / \mathrm{dia}$. Além desse medicamento, a paciente fazia uso de Carbonato de Cálcio + Vit D; e Metoprolol 100mg/dia (tratamento anti-hipertensivo).

Quadro 1 - Laudo com o resultado do Holter realizado na paciente em 24 de junho de 2019.

\begin{tabular}{|l|}
\hline \multicolumn{1}{|c|}{ HOLTER DE 24 HORAS } \\
\hline $\begin{array}{l}\text { 1. Ritmo sinusal alternado com o de fibrilação atrial, sustentada, não sustentada, ora com } \\
\text { condução normal, ora com aberrância e com elevada resposta ventricular (206 bpm); }\end{array}$ \\
\hline 2. Ausência de pausas maiores de 2,5 segundos; \\
\hline $\begin{array}{l}\text { 3. A frequência cardíaca variou de } 52 \text { até } 92 \text { batimentos por minuto (bpm), com média de } 84 \\
\text { bpm; }\end{array}$ \\
\hline $\begin{array}{l}\text { 4. Presença de extrassístoles supraventriculares frequentes (45129 ESV): } 42227 \text { ESV } \\
\text { isoladas, 1020 ESV pareadas, bigeminadas (755 eventos) e trigeminadas (276 eventos); }\end{array}$ \\
\hline 5. Condução atrioventricular normal; \\
\hline 6. A condução intraventricular não se modificou no decorrer do exame; \\
\hline 7. Presença de extrassístoles ventriculares isoladas, monomórficas e raras (2 EV); \\
\hline 8. Ausência de alterações na repolarização ventricular; \\
\hline 9. Intervalo QT normal; \\
\hline 10. Diário elucidativo. Não houve relato de sintomas. \\
\hline
\end{tabular}

Fonte: Silva SS, et al., 2021.

Tabela 1 - Ecocardiograma realizado em 26 de junho de 2019, demonstrando aumento importante dos átrios, função sistodiastólica do VE normal e esclerose valvar aórtica sem repercussão hemodinâmica.

\begin{tabular}{lcc}
\hline Parâmetros estruturais & $33 \mathrm{~mm}$ & $20 \mathrm{a} 37 \mathrm{~mm}$ \\
\hline Aorta (Diâmetro da raiz) & $38 \mathrm{~mm}$ & $20 \mathrm{a} 40 \mathrm{~mm}$ \\
Átrio Esquerdo & $18 \mathrm{~mm}$ & $07 \mathrm{a} 26 \mathrm{~mm}$ \\
Diâmetro Ventrículo Direito & $52 \mathrm{~mm}$ & $35 \mathrm{a} 56 \mathrm{~mm}$ \\
Diâmetro Diastólico Final do Ventrículo Esquerdo ( VE) & $33 \mathrm{~mm}$ & $25 \mathrm{a} 40 \mathrm{~mm}$ \\
Diâmetro Sistólico Final do VE & $18 \mathrm{~mm}$ & 07 a $11 \mathrm{~mm}$ \\
Espessura Diastólica do Septo & $08 \mathrm{~mm}$ & 07 a $11 \mathrm{~mm}$ \\
Espessura Diastólica Parede Posterior do Ventrículo Esquerdo (PPVE) & & \\
\hline Relações e Funções Ventricluares & 1,15 & $1,0 \pm 0,5$ \\
\hline Relação Átrio Esquerdo / Aorta & $66 \%$ & $>58 \%$ \\
Fração de Ejeção (Teicholz) & $146 \mathrm{~g}$ & $94 \mathrm{a} 276 \mathrm{~g}$ \\
Massa Ventricular Esquerda & $87,91 \mathrm{~g} / \mathrm{m}^{2}$ & \\
Relação Massa / Superfície Corporal & $37 \%$ & - \\
Percent Encurt Cavidade & 1,00 & $<1,3$ \\
Relação Septo / PPVE & $130 \mathrm{ml}$ & $73 \mathrm{a} 156 \mathrm{ml}$ \\
Volume Diastólico Final & $85 \mathrm{ml}$ & $54 \mathrm{a} 99 \mathrm{ml}$ \\
Volume Sistólico & $0,96 \mathrm{ml} / \mathrm{g}$ & $0,45 \mathrm{a} \mathrm{0,9} \mathrm{ml/g}$ \\
Relação Volume / Massa & $44 \mathrm{ml}$ & $18 \mathrm{a} 57 \mathrm{ml}$ \\
Volume Sistólico Final & &
\end{tabular}

Fonte: Silva SS, et al., 2021. 
Figura 1 - Eletrocardiograma onde se observa 71 bpm, ritmo irregular. Eixo: $0+30$.

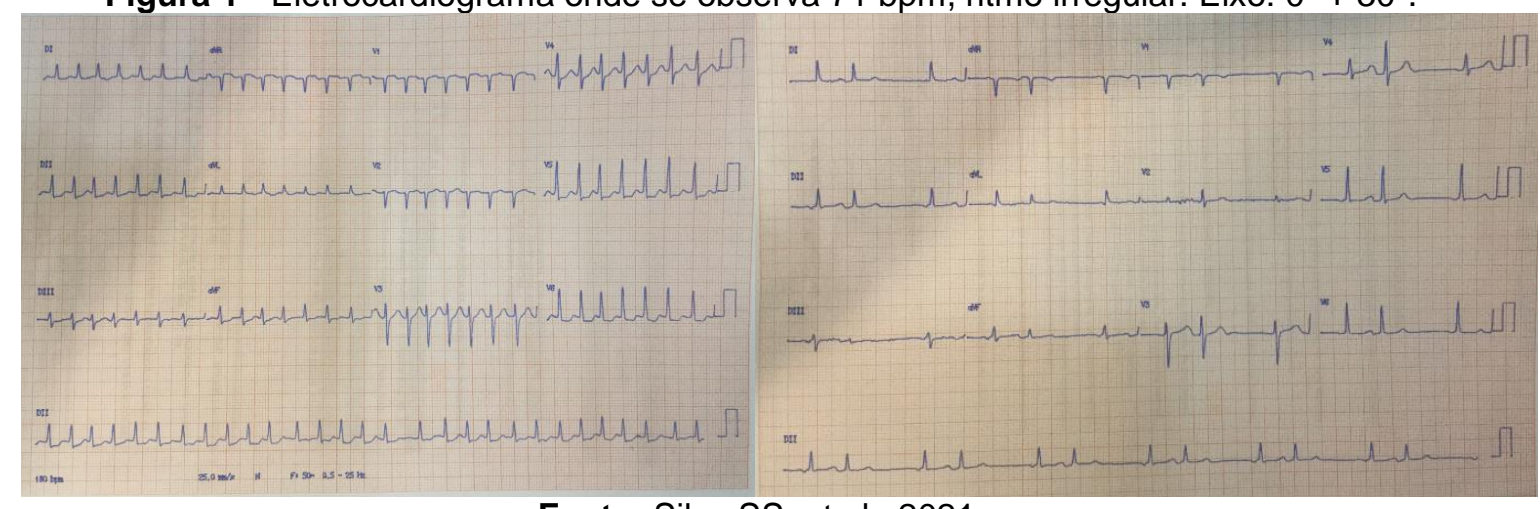

Fonte: Silva SS, et al., 2021.

Em exame clínico no dia 26 de julho foi constatada dor abdominal epigástrica com irradiação retroesternal e dispneia aos pequenos esforços com melhora ao decúbito lateral, com ausência de febre, hidratada e hipocorada. Em exame do tórax foi verificada expansibilidade aumentada e: Ausculta cardíaca: ritmo cardíaco irregular, bulhas normofonéticas, sem sopros; Frequência cardíaca: 49 batimentos por minuto; Ausculra respiratória: murmúrio vesicular fisiológico, sem ruídos adventícios. Frequência respiratória: 22 incursões respiratórias por minuto. Saturação periférica de oxigênio: 96\% em ar ambiente. Paciente negou tabagismo, etilismo ou uso de outras drogas. Verificada leucocitose importante $(>200.000)$, em exames em departamento de urgência.

A paciente foi internada para investigação diagnóstica para: síndrome infecciosa, síndrome paraneoplásica, síndrome mieloproliferativa e/ou síndrome de Gaucher. Entre as suspeitas estavam: reação leucemoide (adenocarcinoma pulmonar, carcinoma papilífero renal); leucemia mieloide aguda (leucostase); leucemia mieloide crônica; doença de Gaucher. Iniciou-se tratamento com Amiodarona (fármaco do grupo dos antiarrítmicos da classe III de amplo espectro e um potente vasodilatador) e Oxacilina $2 \mathrm{~g}$ por via endovenosa (EV) de quatro em quatro horas (4/4h).

No dia 28 de julho iniciou um quadro de: Hipotensão; taquiarritmia; dispneia e hipóxia com necessidade de suporte de oxigênio; sonolência e confusão mental; aumento do padrão da temperatura com picos febris (38,6 graus centígrados). Foram aventadas as hipóteses de pneumonia e leucostase.

Foi iniciada antibioticoterapia com Tazocin ${ }^{\circledR}$ (piperacilina sódica, tazobactam sódico) e Vancomicina, pensando-se em uma possível pneumonia (confirmada em radiografia do tórax realizada em 29 de julho de 2019 - Figura 2) gerando choque séptico e instabilidade hemodinâmica, porém sem melhora após o $4^{\circ}$ dia de tratamento.

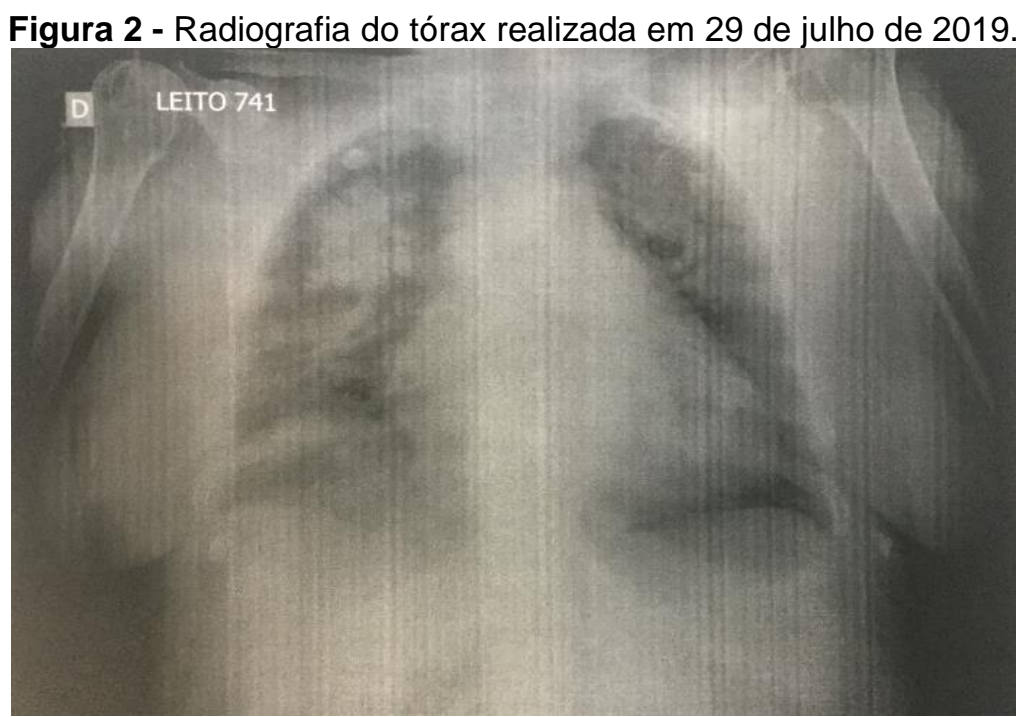

Fonte: Silva SS, et al., 2021. 
A paciente só obteve melhora do quadro clínico após a realização da citorredução com hidroxiureia, com diminuição da contagem de leucócitos para 50.000. Assim chegou-se ao diagnóstico clínico de leucostase, secundária a leucemia mieloide aguda, com comprometimento pulmonar importante e neurológico leve, com melhora após citorredução com hidroxiureia.

A paciente seguiu com o seguinte tratamento: dieta oral hipossódica; Vancomicina um grama (1g) EV 12/12h; Tazocin 4,5g EV de 6/6h; Carvedilol 3,125 miligramas (mg) via oral (VO) de 12/12h; Enalapril 5mg 1 vez ao dia; Espironolactona 25mg 1x/dia; Furosemida 40mg VO 1 vez ao dia; Xarelto 15mg VO 1x/dia; Alopurinol 100mg VO de 8/8h; Hidroxiureia $1 \mathrm{~g}$ VO de 8/8h.

\section{DISCUSSÃO}

Segundo Tendulkar AA, et al. (2017), a LMA pode apresentar leucostase em contagens de leucócitos acima de $100 \times 109 / \mathrm{L}$ com sintomas graves, afetando principalmente o sistema nervoso central e o sistema respiratório, conforme se constatou na paciente do caso aqui em comento. De acordo com estes autores, as medidas adequadas para o manejo dessa emergência médica incluem hidratação, citorredução, manejo de SLT e redução terapêutica de leucócitos (TLR), especialmente em casos complicados por leucostase e síndrome de hiperviscosidade. A justificativa por trás da realização de TLR reside no fato de que influencia a diferenciação celular na medula óssea, aumentando a proporção de células blásticas na fase $\mathrm{S}$. Isso se traduz em maior eficiência de certos agentes antineoplásicos, como a citarabina e o metotrexato.

Como a Sociedade Americana de Aférese (ASFA) apóia a implementação de rotina de TLR em casos de LH secundária a LMA com sinais de leucostase, Tendulkar AA, et al. (2017) estudarama segurança e eficácia de TLR como uma estratégia de leucorredução em pacientes que apresentam HL com características de leucostase, sintomas de hiperviscosidade e SLT. Foram acompanhados por quatro anos uma série de casos de dez pacientes que realizaram TLR, juntamente com outras medidas de suporte. A sobrevida média em quatro anos de acompanhamento foi de 12,8 meses e a mortalidade geral foi de $20 \%$. Os autores concluíram que a TLR é uma terapia segura e eficaz para leucorredução.

No caso de presente estudo optou-se pela citorredução com hidroxiureia, que também foi utilizada no estudo realizado na Rússia por Troitskaya VV, et al. (2015), onde os autores avaliaram a eficácia da política de tratamento de pacientes com LMA e LH, que visa prevenir a síndrome da hipercitólise rápida e lise tumoral maciça (citólise) e/ou reduzir o grau desta última no início da poliquimioterapia de indução. Para realizar o estudo, os autores utilizaram o período de 2010 a 2014, no Centro de Pesquisa em Hematologia do Ministério da Saúde da Rússia, que tratou 92 pacientes com LMA, dos quais 18 pacientes apresentavam LH no início da doença. Todos os examinados receberam terapia citorredutora com hidroxiureia e, na presença de leucostase e/ou leucocitose, com leucaferese. Em caso de redução da leucocitose, foi realizada plasmaférese para prevenir (tratar) a citólise. Foi aplicada daunorrubicina nos dias 3-5 do ciclo de indução $7+3$.

Os resultados do estudo de Troitskaya VV, et al. (2015) demonstraram que os sinais de leucostase foram detectados em mais da metade dos 18 pacientes com maior contagem de leucócitos: 13 (72\%) com lesão pulmonar, incluindo 5 deles com sinais de síndrome do desconforto respiratório. Foi realizada terapia de citorredução com hidroxiureia com dez miligramas por quilo, por dia. A taxa de remissão foi de $89 \%$, sobrevida global e livre de recidiva em três anos foram de $50 \%$. Com isso, concluiu-se que terapias citorredutivas adequadas podem prevenir a síndrome de citólise tumoral maciça.

Segundo Thapa N, et al. (2018), o início da quimioterapia sem redução prévia da leucocitorredução pode levar à SLT. Nesse caso, a leucaferese terapêutica (LT) é usada como uma intervenção leucocitorredutora. Para averiguar sua segurança para paciente pediátricos, estes autores fizeram uma revisão retrospectiva dos prontuários de 19 pacientes. Os resultados identificaram uma sobrevida em curto prazo imediatamente após a LT foi de $100 \%$, sem qualquer complicação importante do procedimento. O tempo médio de sobrevida em pacientes com LMA foi de 1,5 anos e com leucemia linfoblástica aguda foi de 6,5 anos. 
Concluíram que a LT reduz significativamente o número de leucócitos em pacientes pediátricos com leucemia a partir dos 22 dias de idade e que não foi associada a nenhuma complicação significativa, o que sugere que a LT é um procedimento inicial seguro na leucemia pediátrica.

Porém, conforme O'Doherty U (2014), apesar de a leucaferese ser usada em um esforço para diminuir rapidamente a contagem de blastos circulantes de um paciente, o que pode prevenir o desenvolvimento de leucostase e fornecer alívio sintomático da leucostase,seu impacto na mortalidade precoce e em longo prazo ainda é controverso, com vários estudos produzindo resultados conflitantes.

O estudo de Piro E, et al. (2011) constatou que a leucaferese associada à quimioterapia em baixas doses reduziu progressivamente os blastos circulantes, melhorando os sintomas respiratórios e limpando os infiltrados pulmonares previamente detectados com a tomografia computadorizada de tórax de alta resolução. O mesmo se aplica aos sintomas neurológicos cuja regressão foi paralela à diminuição de leucócitos. A abordagem foi segura e provavelmente decisiva no manejo do quadro de emergência, permitindo a posterior administração da quimioterapia de indução padrão

Em estudo mais recente, desenvolvido por Stahl M, et al. (2020), coletaram retrospectivamente dados de pacientes com LMA recém-diagnosticada que apresentaram $\mathrm{HL}$ em 12 centros nos Estados Unidos e Europa de 2006 a 2017 e receberam quimioterapia intensiva. Entre 779 pacientes, leucostase clínica foi relatada em $27 \%$, e leucaferese foi usada em 113 pacientes (15\%). A mortalidade em 30 dias foi de 16,7\%. Entre os pacientes com leucostase clínica julgada pelo investigador, houve melhorias estatisticamente significativas na mortalidade em 30 dias. Entretanto, dado o uso significativo de recursos, custo e complicações potenciais da leucaferese, estudos randomizados são necessários para avaliar seu valor.

A LMA pode resultar em insuficiência respiratória aguda (IRA) nos primeiros dias, exigindo internação em unidade de terapia intensiva (UTI) em metade dos casos.Há três síndromes específicas da leucemia responsáveis pela IRA: leucostase, infiltração leucêmica pulmonar (ILP) e pneumopatia por lise aguda (PLA). Estas três síndromes resultam em IRA rapidamente progressiva. Em pacientes com IRA no estágio inicial da LMA, o desafio clínico consiste em identificar rapidamente o mecanismo ou mecanismos subjacentes e selecionar as melhores opções de tratamento. Leucaferese e hidroxiureia foram propostas como terapias para melhorar a disfunção orgânica relacionada à leucostase, mas seu uso permanece controverso. Mais recentemente, foi relatado que a terapia com esteroides melhora a função respiratória e a mortalidade em pacientes com LMA de alto risco (MOREAU AS, et al., 2014).

Diante disso, Moreau AS, et al. (2014) analisaram retrospectivamente os dados clínicos e laboratoriais de 114 pacientes internados em uma UTI médica dentro de 10 dias após o diagnóstico de LMA. Os eventos respiratórios (ERs) ocorreram em 95 pacientes e eram específicos da leucemia em 58 pacientes (61\%). A mortalidade no dia 28 foi de $34,5 \%$ em pacientes com ERs específicos para leucemia (leucostase, $41 \%$; ILP, $23 \%$; e PLA, $31 \%$ ) e $48,6 \%$ em pacientes com outros ERs. Por análise multivariada, os fatores de risco independentes para morte foram idade $>50$, status do Eastern Cooperative Oncology Group (ECOG) $\geq 2$ e necessidade de ventilação mecânica. Os autores constataram que a terapia com dexametasona se mostrou protetora, sugerindo um papel importante como tratamento preventivo em pacientes com envolvimento pulmonar não infeccioso relacionado à LMA.

Cabe destacar que a leucostase, também chamada de hiperleucocitose sintomática, que foi apresentada pela paciente deste relato de caso, é uma emergência médica, mais comumente observada em pacientes com LMA.É caracterizada por uma contagem de células blásticas muito alta, gerando uma diminuição da perfusão tecidual (> 100.000 células) (AMÂNCIO J, et al., 2008).Pode apresentar-se em um quadro de desconforto respiratório progressivo, podendo estar associado a um quadro neurológico (MOREAU AS, et al., 2014), conforme ocorrido no caso aqui relatado.

Quando não precocemente diagnosticada pode levar ao óbito, como ocorrido no estudo de Goenka $P$, et al. (1992), que relatam o caso de uma paciente de 75 anos com leucemia mieloide crônica em fase acelerada e hiperleucocitose, que apresentou síndrome do desconforto respiratório agudo. Apesar do 
suporte pulmonar precoce e agressivo e da quimioterapia citorredutora, a paciente morreu. Na autópsia foi confirmada a presença da síndrome de leucostase. Da mesma forma, no estudo de Mahave MCV, et al. (1999), dois pacientes com LMA que desenvolveram uma síndrome do desconforto respiratório por leucostase foram a óbito.

Por fim, conforme alega Korkmaz S (2018), medidas como hidroxiureia, quimioterapia de baixa dose e leucaferese não devem ser consideradas para corrigir as anormalidades laboratoriais em pacientes com HL assintomáticos. O tratamento ideal da HL sintomática ainda é incerto e não há estudos randomizados que demonstrem que um é superior ao outro. Portanto, recomenda-se que a quimioterapia intensiva seja implementada o mais rápido possível em pacientes elegíveis ao tratamento, em paralelo com medidas de suporte para CID e SLT.

Com base na literatura atual disponível acerca da leucostase secundária a hiperleucocitose em pacientres com Leucemia Mielóide Aguda, conclui-se que é de fundamental importância o conhecimento mais aprofundado dessa entidade clínica caracterizada como uma emergência médica. Dessa forma, o conhecimentodas manifestação clínicas clássicas para o seu diagnóstico e tratamento precoces são essenciais, visto que podem impactar de forma importante na diminuição da mortalidade desses pacientes.

\section{REFERÊNCIAS}

1. AMÂNCIO J, et al. Leucemia mielóidecrônica e síndrome de hiper-viscosidade. Relato de caso. RevistaBrasileira de Terapialntensiva, 2008; 20(1): 99-102.

2. AQUI N, O'DOHERTY U. Leukocytapheresis for the treatment of hyperleukocytosis secondary to acute leukemia. Hematology. American Society of Hematology. Education Program. 2014; 2014(1): 457-460.

3. AZOULAY É, et al. Dexamethasone in patients with acute lung injury from acute monocytic leukaemia. The European Respiratory Journal. 2012; 39(3): 648-653.

4. BEWERSDORF JP, ZEIDAN AM. Hyperleukocytosis and Leukostasis in Acute Myeloid Leukemia: Can a Better Understanding of the Underlying Molecular Pathophysiology Lead to Novel Treatments? Cells. 2020; 9(10): 2310.

5. CHAOUI D, et al. Incidence and prognostic valueof respiratory events in acute leukemia. Leukemia 2004; 18: 670675.

6. GOENKA P, et al. Acute leukostasis pulmonary distress syndrome. The Journal of Family Practice. 1992; 35(4): 445449.

7. KORKMAZ S. The management of hyperleukocytosis in 2017: Do we still need leukapheresis? Transfusion and Apheresis Science. 2018; 57(1): 4-7.

8. MAHAVE MCV, et al. Síndrome de distrésrespiratorio por leucemiashiperleucocíticas. Anales de Medicina Interna (Madrid). 1999; 16(7): 359-360.

9. MOREAU AS, et al. Respiratory events at the earliest phase of acute myeloid leukemia. Leukemia \&Lymphoma, 2014; 55(11): 2556-2563.

10. PIRO E., et al. Reversal of leukostasis-related pulmonary distress syndrome after leukapheresis and low-dose chemotherapy in acute myeloid leukemia. Journal of Clinical Oncology. 2011; 29(26): 725-726.

11. STAHL M, et al. Management of hyperleukocytosis and impact of leukapheresis among patients with acute myeloid leukemia (AML) on short- and long-term clinical outcomes: a large, retrospective, multicenter, international study. Leukemia, 2020; 34(12): 3149-3160.

12. TENDULKAR AA, et al. Therapeutic leukocyte reduction for acute and chronic myeloid leukemias: A 4-year experience from an oncology center in India. Asian journal of transfusion science. 2017; 11(2): 156-161.

13. THAPA N., et al. Therapeutic leukocytapheresis in infants and children with leukemia and hyperleukocytosis: A single institution experience. Journal of Clinical Apheresis. 2018; 33(3): 316-323.

14. TROITSKAYA VV, et al. [Treatment in adult patients with acute myeloid leukemias and hyperleukocytosis at disease onset]. Terapevticheskiiarkhiv. 2015; 87(7): 33-40.

15. VINCENT $F$, et al. Priseen charge thérapeutique des manifestations pulmonaires à la phase aiguë des leucémiesaiguësmyéloblastiques. Revue des Maladies Respiratoires. 2012; 29(6): 743-55. 